\title{
Brain activation of eye movements in subjects with refractive error
}

This article was published in the following Dove Press journal:

Eye and Brain

14 May 2010

Number of times this article has been viewed

\section{Gereon Nelles' \\ Anja Pscherer' \\ Armin de Greiff ${ }^{2}$ \\ Joachim Esser ${ }^{3}$}

'Neurologische Klinik und Poliklinik, Universitätsklinikum Essen; ${ }^{2}$ Institut für Diagnostische und Interventionelle Radiologie und Neuroradiologie, Universitätsklinikum Essen;

${ }^{3}$ Zentrum für Augenheilkunde, Abteilung für Erkrankungen des vorderen Augenabschnittes, Universitätsklinikum Essen
Correspondence: Gereon Nelles Werthmannstr Ic, D-50935 Köln, Germany

$\mathrm{Tel}+492219438120$

Fax +4922194381229

Email gereon.nelles@uni-essen.de
Purpose: Recent functional magnetic resonance imaging (fMRI) studies have described reorganized activation of the oculomotor and visual cortex after focal brain lesions. These studies are based on comparison with healthy individuals who may have a very heterogenous refractive error. The influence of refractive error on the cortical control of an oculomotor task such as a prosaccade trial, however, is unknown.

Methods: To investigate the influence of visual acuity on changes of cortical oculomotor control, we studied the representation of visually guided prosaccades in nine subjects with refractive error and 11 normally sighted subjects using fMRI. Correction of refractive error was not allowed during fMRI. Differences in activation between rest and saccades as well as between subjects with refractive error vs subjects with normal vision were assessed with statistical parametric mapping.

Results: In both groups, activation of a frontoparietal network was observed. Subjects with refractive errors showed increased activation compared to normally sighted subjects, with overactivation in bilateral frontal and parietal eye fields, supplementary eye fields, as well as in the bilateral extrastriate cortex.

Conclusions: This group of subjects with refractive error showed increased activation in an extended oculomotor and visual network to maintain performance during simple prosaccades. This observation underlines the importance of using appropriate control groups in fMRI-studies after brain lesions.

Keywords: eye movement, functional magnetic resonance imaging (fMRI), refractive error, saccades, vision

\section{Introduction}

Functional magnetic resonance imaging (fMRI) is increasingly used to study the pathophysiology of the oculomotor system. In healthy individuals, the areas that control eye movements are located in the frontal and parietal cortex and in subcortical structures. In addition, the occipital cortex is active when subjects perform saccades, suggesting that parallel activity of the motor and primary visual sensory areas is necessary to perform voluntary eye movements. The cerebral representation of saccades has been well characterized in electrophysiological experiments of primates, ${ }^{1}$ functional imaging studies of healthy individuals, ${ }^{2,3}$ and in patients with unilateral lesions of the frontal or parietal cortex. ${ }^{4}$ Furthermore, visual field defects after striate lesions are associated with changes in the frontoparietal network underlying the cortical control of saccades. ${ }^{5}$

First symptoms of refractive error may occur at any age. Symptoms include difficulty reading fine print particularly in low light conditions, eyestrain when 
reading for long periods, blur at near vision, or momentarily blurred vision when transitioning between viewing distances. During daily living activities, the reduced ability to focus is compensated by corrective lenses including eyeglasses or contact lenses.

If fMRI-studies of the visual system are performed without corrective lenses, irrespective of the subjects' refractive status, imaging results may be confounded. In subjects with reduced visual acuity due to macular degeneration, for example, increased activation of the prefrontal and parietal cortex has been shown. ${ }^{6}$ Subjects with decreased visual acuity as a result of refractive error may have similar changes of cortical control of eye movements. Therefore, in this study, we compared the cortical networks that underlie oculomotor function in patients with reduced visual acuity with those in normally sighted control subjects. We hypothesized that activation of a frontoparietal network would be observed in subjects with refractive error compared to normally sighted subjects.

\section{Methods}

\section{Subjects}

Nine subjects with refractive error and eleven normally sighted subjects were studied. Subjects without refractive errors were aged between 23 and 66 years (mean $38 \pm 14$ years) and had visual acuity without correction of $20 / 20$ or better. Subjects with decreased visual acuity were aged between 25 and 70 (mean $52 \pm 14$ years). Their refractive status is summarized in Table 1. All subjects had no history of neurological or psychiatric disease. Prior to each fMRI, a full neurological exam was obtained for each subject. Data of vision status was provided by subjects' ophthalmologists. All subjects gave written informed consent. This study was approved by the Review Board of Ethics Committee of the University of Essen.

Table I Refractive status of subjects using correction of refractive error in daily life

\begin{tabular}{lll}
\hline Subject & Right & Left \\
\hline 1 & +3.25 & +1.75 \\
2 & +6.0 & +4.5 \\
3 & -2.0 & -2.25 \\
4 & -3.0 & -2.25 \\
5 & -5.5 & -4.25 \\
6 & -2.0 & -3.0 \\
7 & -0.5 & -1.5 \\
8 & -3.0 & -3.25 \\
9 & -2.0 & -2.25 \\
\hline
\end{tabular}

\section{Tasks}

We used a block design with alternating rest and activation tasks. Visual stimuli were back-projected with an LCDprojector onto a $154 \times 115 \mathrm{~cm}^{2}$ screen placed $90 \mathrm{~cm}$ in front of the magnet bore. The light intensity of the background screen was kept constant during baseline and stimulation in order to avoid unintentional stimulation caused by changes in brightness of the environment. Luminance of the projector was 1100 ANSI-Lumen with 400:1 contrast.

\section{Fixation}

Subjects were instructed to maintain fixation on a green point in the center of the screen. The fixation point was constantly illuminated. No peripheral stimuli were presented. The distance between the eyes and the fixation point was $90 \mathrm{~cm}$.

\section{Saccades}

A green point appeared at the center, then randomly at $21^{\circ}$ of eccentricity to either the left or right from the center, and again at the center of the screen (presentation time, $750 \mathrm{msec}$ ). Subjects were asked to follow the visual cue with horizontal saccades. All subjects were experienced in performing the saccade trial and practiced repetitively in the laboratory. All subjects were adapted to the scanner and the task in a prescan session. During fMRI, subjects did not wear contact lenses or spectacles.

\section{Oculomotor recordings}

Fixation and saccades were monitored using MRI-compatible bipolar and bidimensional semiquantitative electrooculographic recordings. The purpose of these recordings was to assess reliability of task performance. Fixation and saccades were well performed in all subjects (Figure 1).

\section{fMRI image acquisition and analysis}

We acquired 130 volumes of 30 axial fMRI images $(1.5 \mathrm{~T}$, $40 \mathrm{mT} / \mathrm{m}$ gradient, $3.5 \times 3.5 \times 3.3 \mathrm{~mm}$ spatial resolution; TR/TE $3000 / 60 \mathrm{~ms}$, flip angle $90^{\circ}$ ). In each subject, seven repetitions of 10 scans during fixation (F) alternating with six blocks of saccades (S) were obtained (FSFSFSFSFSFSF) yielding a total of 70 volumes for fixation and 60 volumes for saccades. Each cycle lasted $30 \mathrm{sec}$. During one cycle of saccades, 15 peripheral targets were presented on each side. Raw data were processed and analyzed using statistical parametric mapping (SPM'99; Wellcome Department of Cognitive Neurology, London, UK). Functional images were realigned with sinc interpolation and then normalized to the standard stereotactic space $\left(3 \times 3 \times 3 \mathrm{~mm}^{3}\right)$ corresponding to the template from 


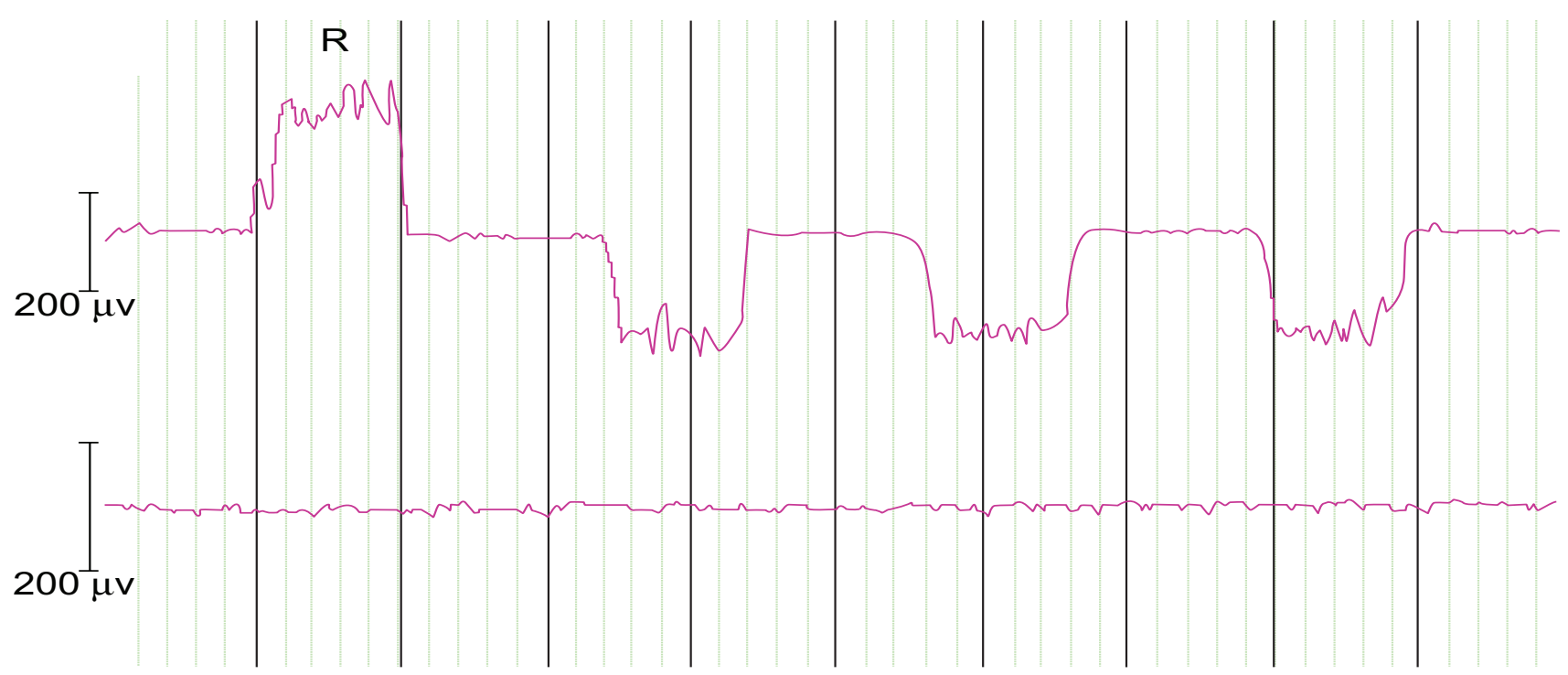

Figure I Electrooculography (EOG) recording during saccades (upper curve) and during fixation (lower curve). EOG was recorded to monitor reliability of task performance while the subject was imaged.

Notes: Interval between two thick lines $=I \mathrm{sec} . \mathrm{R}=$ saccade to the right.

the Montreal Neurological Institute (see http://www.mrc-cbu. cam.ac.uk/Imaging/mnispace.html). Functional images were smoothed to accommodate intersubject variability of brain anatomy using an isotropic Gaussian kernel of $9 \mathrm{~mm}$. Data were modeled using a box-car function convolved with the hemodynamic response function and its temporal derivative.

\section{Statistics}

Assessment of significant signal changes between rest and visual stimulation was performed using statistical parametric mapping. ${ }^{7}$ A voxel-by-voxel comparison according to the general linear model and $t$-test statistics were used to calculate differences of activation between saccades and rest. The same model was used for comparisons between subjects with impaired visual acuity vs subjects with normal vision (multigroup study). Statistical inferences were based on group comparison using a fixed effects model. ${ }^{8}$ The resulting statistical parametric map (SPM) was subsequently used to assign $P$-values (to voxels and also to clusters), which were corrected for multiple comparisons applied for the whole brain $(P<0.001)$. The locations of activation maxima were identified with reference to the atlas of Talairach and Tournoux. ${ }^{9}$

\section{Results}

\section{Saccades versus fixation in normally sighted subjects}

Most significant areas of activation were found bilaterally in the frontal eye field. The maximum coincided with the posterior bank of the precentral gyrus in Brodmann area
6 between 50 and $61 \mathrm{~mm}$ above the anterior posterior commissural (ACPC) line. Brodmann area 6 was also activated at the dorsomedial wall of the frontal lobe and above the cingulate sulcus, corresponding to the supplementary eye field (coordinates $3,-3,61$ ). Bilateral activation occurred in the parietal eye fields of Brodmann area 7, and also in the striate and extrastriate cortex. Table 2 summarizes the areas of maximum activation in normally sighted subjects. Areas of activation are shown in Figure 2.

\section{Saccades versus fixation in subjects with refractive errors}

Activation was more significant in all areas in subjects with refractive errors compared to normally sighted subjects.

Table 2 Areas with significant activation during saccades in normally sighted subjects

\begin{tabular}{llll}
\hline Localization & BA & $\mathbf{x}, \mathbf{y}, \mathbf{z}$-coordinates & $\mathbf{z}$-score \\
\hline FEF right & 6 & $45,-1,50$ & 12.4 \\
FEF left & 6 & $-42,-3,50$ & 12.0 \\
SEF & 6 & $3,-3,61$ & 11.6 \\
PEF right & 7 & $33,-47,47$ & 7.5 \\
PEF left & 7 & $-27,-53,52$ & 12.2 \\
Striate cortex right & $17 / 18$ & $12,-84,4$ & 12.0 \\
Striate cortex left & $17 / 18$ & $-9,-84,7$ & 10.5 \\
Extrastriate cortex right & $19 / 18$ & $15,-64,-4$ & 13.7 \\
Extrastriate cortex left & 19 & $-12,-73,-9$ & 14.0 \\
\hline
\end{tabular}

Notes: Group study, $P<0.00$ I, corrected. Coordinates according to Talairach and Tournoux. ${ }^{9}$

Abbreviations: BA, Brodmann area; FEF, frontal eye fields; PEF, parietal eye fields; SEF, supplementary eye fields. 

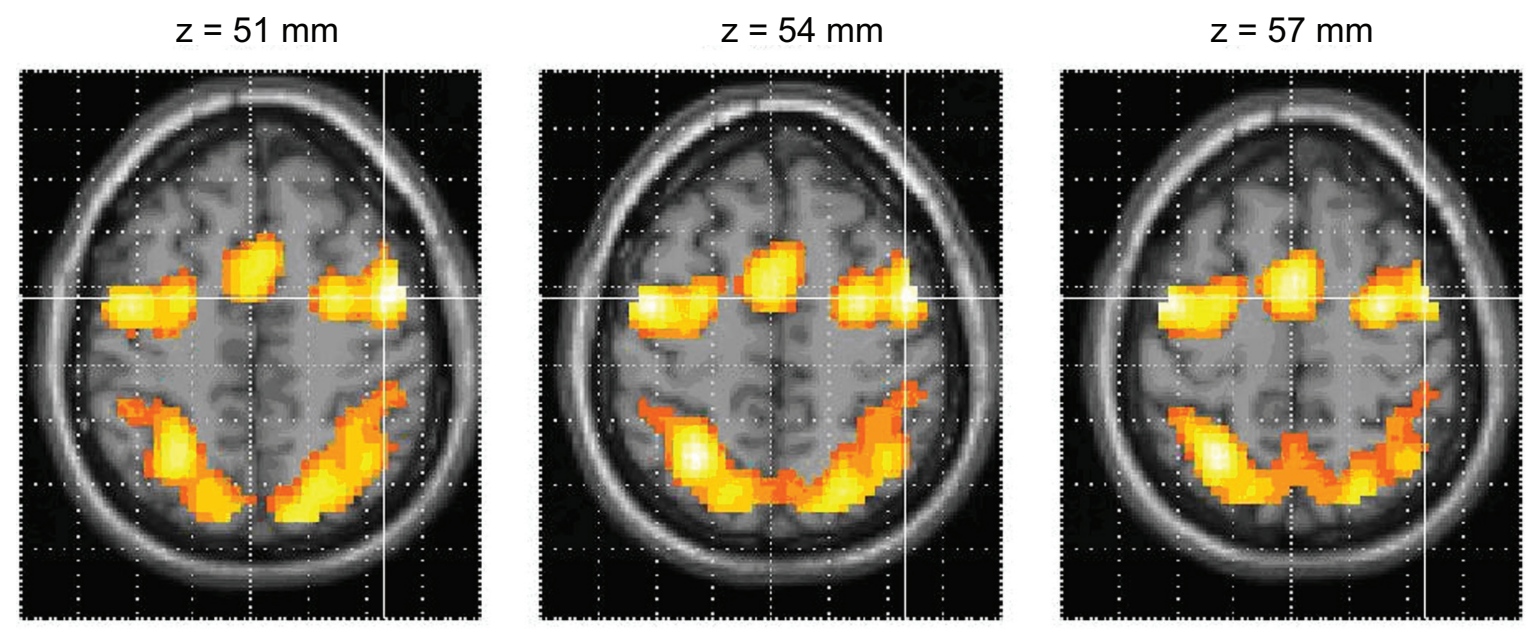

T value

Notes: Group study; $P<0.001$. See Table 2 for all anatomic coordinates.

Abbreviation: SPM, statistical parametric map.

Most significant areas of activation were again found in frontal eye fields, in the supplementary eye field, in parietal eye fields, and in the extrastriate cortex (Table 3, Figure 3).

\section{Comparison of activation areas in subjects with refractive errors compared to normally sighted subjects}

Differences in the activation pattern during saccades between normally sighted subjects and subjects with refractive error were identified by subtracting significant regions of activation of normally sighted subjects from those with

Table 3 Increases of neuronal activation during saccades in subjects with decreased visual acuity

\begin{tabular}{llll}
\hline Localization & BA & $\mathbf{x}, \mathbf{y}, \mathbf{z}$-coordinates & z-score \\
\hline FEF right & 6 & $24,-5,58$ & 20.7 \\
FEF left & 6 & $-39,2,50$ & 14.1 \\
SEF & 6 & $0,3,61$ & 21.0 \\
PEF right & 7 & $24,-55,59$ & 20.7 \\
PEF left & 7 & $-39,-53,47$ & 9.8 \\
Striate cortex right & 17 & $12,-87,-1$ & 19.4 \\
Striate cortex left & 17 & $-6,-94,2$ & 15.7 \\
Extrastriate cortex right & $19 / 18$ & $15,-76,1$ & 13.2 \\
Extrastriate cortex left & 19 & $-15,-76,-4$ & 12.0 \\
\hline
\end{tabular}

Notes: Group study, $P<0.00$ I, corrected. Coordinates according to Talairach and Tournoux. 9

Abbreviations: BA, Brodmann area; FEF, frontal eye fields; PEF, parietal eye fields; SEF, supplementary eye fields. refractive error using a two-sample $t$-test. In this analysis, overactivation in bilateral parietal eye fields and frontal eye fields, the supplementary eye-fields as well as in the bilateral extrastriate cortex were found in subjects with refractive error (Table 4, Figure 4).

\section{Discussion}

In this study, brain activation patterns during visually guided saccades of subjects with refractive errors and normally sighted subjects were compared. In both groups, these saccades were associated with activation of a frontoparietal network. These findings are consistent with the view that saccades in humans are primarily triggered by eye fields in the posterior parietal cortex and frontal cortex. ${ }^{10}$ In both groups, the most significant activation was observed in Brodmann area 6, on the posterior bank of the precentral gyrus between 50 and $61 \mathrm{~mm}$ above the ACPC line. This cluster of activation anterior and lateral to the precentral sulcus coincides with the location of the human frontal eye field. ${ }^{11}$ Activation of this area during visually guided saccades corresponds well to previous studies using positron emission tomography ${ }^{2}$ and fMRI. ${ }^{3}$ In this pilot study, we used a fixed effects model. This model only supports inferences about the group of subjects we looked at, not to the population at large.

Additional activation of the frontoparietal network during performance of a simple prosaccade trial was found in subjects with refractive error compared to normally sighted subjects. Several mechanisms may explain the increased activation in subjects with reduced visual acuity. The increased activation pattern compared to normally sighted 
$\mathrm{z}=51 \mathrm{~mm}$

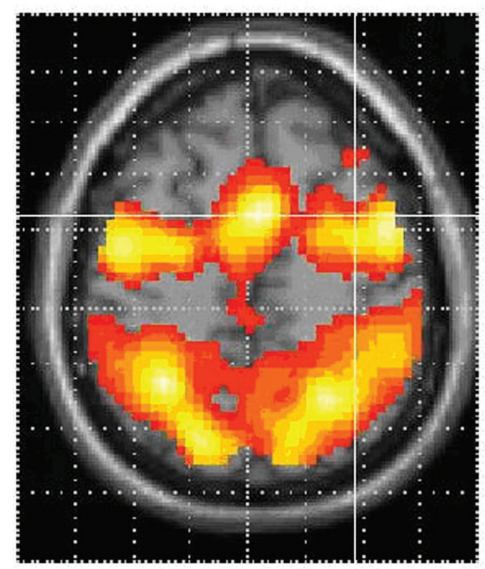

$z=54 \mathrm{~mm}$

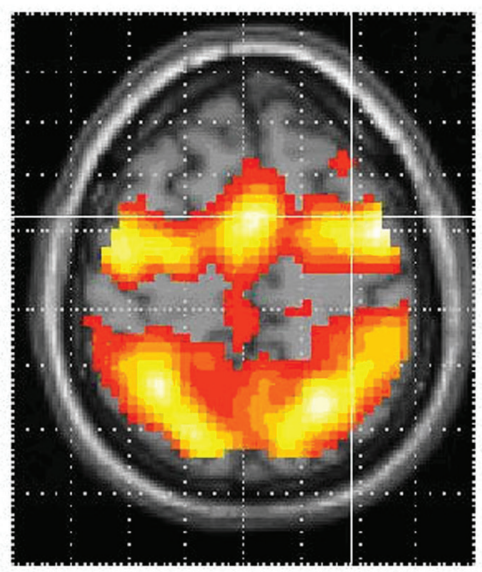

$\mathrm{z}=57 \mathrm{~mm}$

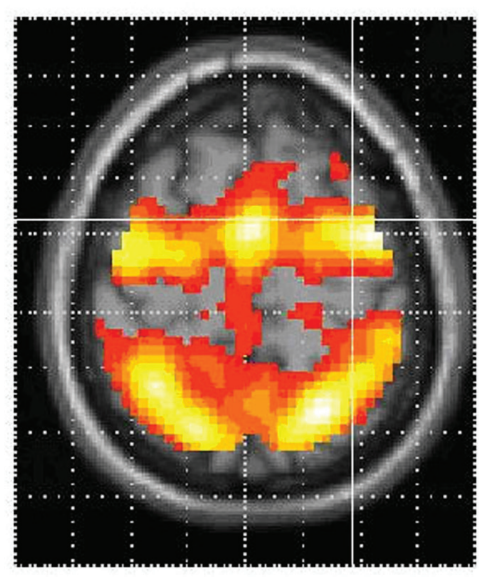

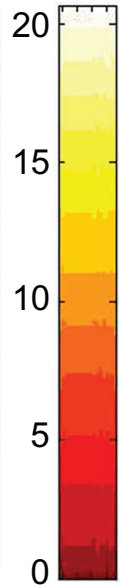

Figure 3 Activation during prosaccade trial in subjects with refractive error. Crosshair shows area with highest z-score.

Notes: Group study; $P<0.001$. See Table 3 for all anatomic coordinates.

Abbreviation: SPM, statistical parametric map.

subjects could suggest recruitment within the frontoparietal network to support neuronal processing and maintain task performance despite reduced visual input. The increased activation in motor and cognitive areas of the oculomotor network, but not in the striate cortex, is consistent with the use of different strategies for locating and following a target. Alternatively, there may also be increased attention and effort during task performance in subjects with refractive error. Performance of eye movement task in subjects with refractive error required greater involvement of cortical regions generally implicated in attention and effort, such as the parietal eye fields. Persistent neural activity of this area has been described during sustained visual attention. ${ }^{12}$

Differences in task performance between both groups could have influenced brain activation patterns. Electrooculogram recordings demonstrated good task performance during fixation and during saccades. These recordings, however,

Table 4 Overactivation in subjects with decreased visual acuity

\begin{tabular}{llll}
\hline Localization & BA & $\mathbf{x}, \mathbf{y}, \mathbf{z}$-coordinates & z-score \\
\hline FEF right & 6 & $39,3,52$ & 7.9 \\
FEF left & 6 & $-39,8,52$ & 6.7 \\
SEF & 6 & $0,3,61$ & 7.7 \\
PEF right & 7 & $27,-55,58$ & 10.3 \\
PEF left & 7 & $-33,-47,55$ & 7.0 \\
Extrastriate cortex right & 19 & $30,-86,26$ & 8.6 \\
Extrastriate cortex left & 18 & $-30,-90,5$ & 8.5 \\
\hline
\end{tabular}

Notes: Multigroup study, $P<0.05$, corrected. Coordinates according to Talairach and Tournoux. ${ }^{9}$

Abbreviations: BA, Brodmann area; FEF, frontal eye fields; PEF, parietal eye fields; SEF, supplementary eye fields. did not permit quantification of numbers or amplitudes of saccades or measurement of onset latency. We are therefore unable to speculate how differences in saccadic behavior could affect the fMRI activation patterns in subjects with refractive error vs normally sighted subjects.

Finally, the increased activation pattern of subjects with impaired vision may also be age-dependent. We have previously shown that older adults increase activation in oculomotor and visual cortical areas during simple prosaccades. ${ }^{13}$ However, in the present study, the age difference between both groups was small. In another study of age-related effects in the oculomotor system, however, Raemaekers and colleagues did not find an increased activation in older subjects. ${ }^{14}$ In fact, they demonstrated an overall reduction of the Blood oxygen level-dependent (BOLD) signal in the visual and oculomotor system of older subjects. We therefore believe that age alone cannot explain the increased oculomotor activation in subjects with impaired vision.

One other study has investigated vision-related differences in cortical activation of eye movements. Little and colleagues investigated brain activation patterns during prosaccade and antisaccade trials in different age groups and in patients with visual impairment due to macular degeneration using event-related fMRI. ${ }^{6}$ In subjects with macular degeneration, the authors demonstrated increased activation of the prefrontal and parietal cortex and a reduction of the BOLD signal in the visual cortex; an observation that corroborates our data. In that study, the oculomotor activation was also not different between younger and older normally sighted subjects. 

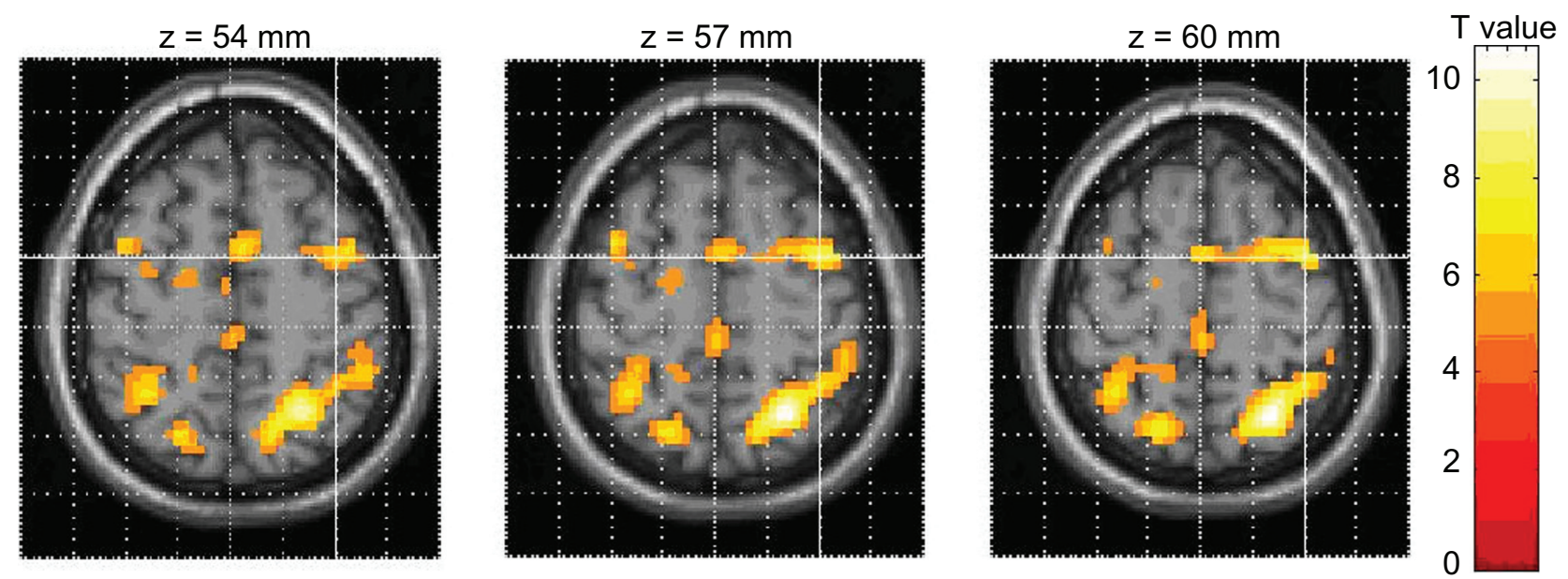

Figure 4 Direct comparisons of subjects with refractive error vs normally sighted subjects. Notes: Multigroup study; $P<0.05$. See Table 4 for all anatomic coordinates.

The effects of refractive error on cortical activation observed in this study adds further evidence to the concept of altered cortical activity in subjects with reduced visual acuity. The present data represent a further step towards understanding the changes of cortical control of eye movements that occur as a result of decreased visual acuity. This observation also underlines the importance of using appropriate control groups in fMRI studies after lesions of the oculomotor system.

\section{Disclosures}

The authors report no conflicts of interest in this work.

\section{References}

1. Bruce CJ, Goldberg ME, Bushnell MC, Stanton GB. Primate frontal eye fields. II. Physiological and anatomical correlates of electrically evoked eye movements. J Neurophysiol. 1985;54:714-734.

2. Anderson TJ, Jenkins IH, Brooks DJ, Hawken MB, Frackowiak RS, Kennard C. Cortical control of saccades and fixation in man. A PET study. Brain. 1994;117:1073-1084.

3. Luna B, Thulborn KR, Strojwas MH, et al. Dorsal cortical regions subserving visually guided saccades in humans: an fMRI study. Cereb Cortex. 1998;8:40-47.
4. Heide W, Kompf D. Combined deficits of saccades and visuo-spatial orientation after cortical lesions. Exp Brain Res. 1998;123:164-171.

5. Nelles G, de Greiff A, Pscherer A, et al. Saccade induced cortical activation in patients with post-stroke visual field defects. $J$ Neurol. 2007;254:1244-1252.

6. Little DM, Thulborn KR, Szlyk JP. An FMRI study of saccadic and smooth-pursuit eye movement control in patients with agerelated macular degeneration. Invest Ophthalmol Vis Sci. 2008;49: 1728-1735.

7. Frackowiak R, Friston KJ, Frith C, Dolan R, Mazziotta J. Statistical inferences. Human Brain Function. San Diego, CA: Academic Press; 1997:85-106.

8. Friston KJ, Holmes AP, Price CJ, Buchel C, Worsley KJ. Multisubject fMRI studies and conjunction analyses. Neuroimage. 1999;10: 385-396.

9. Talairach J, Tournoux P. Coplanar stereotaxic atlas of the human brain. New York, NY: Thieme Verlag; 1988:37-84.

10. Pierrot-Deseilligny C, Rivaud S, Gaymard B, Muri R, Vermersch AI. Cortical control of saccades. Ann Neurol. 1995;37:557-567.

11. Paus T. Location and function of the human frontal eye-field: a selective review. Neuropsychologia. 1996;34:475-483.

12. Srimal $R$, Curtis $C E$. Persistent neural activity during the maintenance of spatial position in working memory. Neuroimage. 2008;39:455-468.

13. Nelles G, de Greiff A, Pscherer A, Esser J. Age related differences of saccade induced cortical activation. Neurosci Lett. 2009;458:15-18.

14. Raemaekers M, Vink M, van den Heuvel MP, Kahn RS, Ramsey NF. Effects of aging on BOLD fMRI during prosaccades and antisaccades. J Cogn Neurosci. 2006;18:594-603.

\section{Publish your work in this journal}

Eye and Brain is an international, peer-reviewed, open access journal focusing on clinical and experimental research in the field of neuroophthalmology. All aspects of patient care are addressed within the journal as well as basic research. Papers covering original research, basic science, clinical and epidemiological studies, reviews and evaluations,

Submit your manuscript here: http://www.dovepress.com/eye-and-brain-journal

\section{Dovepress}

guidelines, expert opinion and commentary, case reports and extended reports are welcome. The manuscript management system is completely online and includes a very quick and fair peer-review system, which is all easy to use. Visit http://www.dovepress.com/testimonials.php to read real quotes from published authors. 